From the Department of Pulmonary and Critical Care and Allergy and Immunology at University Hospitals Cleveland Medical Center in Ohio

(Drs Shilian, Jhaveri, and Hostoffer); Allergy/lmmunology

Associates in Mayfield

Heights, Ohio (Drs Jhaveri and Hostoffer); and the Department of Internal Medicine at University Hospitals Regional Hospitals in Cleveland, Ohio (Dr Wynbrandt); Pulmonary Allergy Critical Care \& Sleep Associates, in Rochester Hills, Michigan (Dr Abraham); and the Department of Pediatrics

Division of Allergy and Immunology at West Virginia University Medicine Children's Hospital in Morgantown (Dr Peppers). This study was presented as a poster at the American Thoracic Society 2018 International Conference in San Diego, California.

Financial disclosures: None reported.

Support: None reported.

Address correspondence to: Ryan Shilian, DO, Department of Pulmonary and Critical

Care and Allergy and Immunology at University Hospitals Cleveland Medical Center, 27155 Chardon Rd, Richmond, OH 44143-1183.

Email: rshilian@gmail.com

Submitted January 25, 2018; revision received April 25, 2019;

accepted May 7, 2019.

\section{Daily Integrated Care Conferences to Reduce Length of Hospital Stay for Patients With Chronic Obstructive Pulmonary Disease}

Ryan Shilian, DO; Tina Abraham, DO; Jonathan Wynbrandt, MD; Devi Jhaveri, DO; Robert W. Hostoffer, DO, LhD, MSMEd; Brian P. Peppers, DO, PhD

Context: Inefficiencies in care coordination — specifically, the lack of an effective method of communication among multiple health care professionals_often leads to an unnecessary increase in length of hospital stay.

Objective: To determine whether daily integrated care conferences (ICCs) would significantly reduce the length of stay for patients with chronic obstructive pulmonary disease (COPD) exacerbation.

Method: Patients with COPD exacerbation were selected for the study using electronic medical records from 2 osteopathic community hospitals located in northeastern Ohio. One hospital used daily ICCs and the other hospital did not use daily ICCs. The average length of stay for patients at each hospital was retrospectively investigated.

Results: A total of 1683 patients with COPD exacerbation were selected. The mean (SD) length of stay in the hospital with daily ICCs was 3.37 (2.89) days compared with 5.55 (3.99) days in the hospital without daily ICCs $(P<.0001)$. At the hospital with daily ICCs, patients aged 40 to 69 years had a $67 \%$ shorter hospital stay and patients aged 70 to 99 years or older had a $36 \%$ shorter length of stay compared with patients at the hospital without daily ICCs.

Conclusion: Daily integrated care conferences significantly reduced the length of stay for patients with COPD exacerbation at an osteopathic community-based hospital. Implementing daily ICCs may make current health care services and coordinated care more efficient, resulting in decreased costs and length of stay for patients with COPD exacerbation.

J Am Osteopath Assoc. 2020;120(3):144-152

doi:10.7556/jaoa.2020.027

Keywords: COPD, integrative care, length of stay

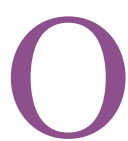

ur current health care system is more complex than ever before. ${ }^{1}$ Today, in order to treat and discharge a patient in a safe and timely manner, a hospital's primary care team often relies on input from various health care providers (eg, primary physician group, pharmacy, respiratory therapy, nutrition, physical and occupational therapy, social work, and case management). Lack of a systematically coordinated method of communication among all of these providers often leads to delayed care. ${ }^{2}$ This, in turn, can cause an unnecessary increase in length of stay, which adds extra costs for patients and health care systems. 
This problem can be alleviated by systematically organizing patient care activities that create a more efficient method of sharing information among all health care providers, which can be accomplished by using care coordination. For example, systematic coordination of care in the form of multidisciplinary rounds (MDRs) is known to reduce the length of hospital stay. ${ }^{3}$ O'Mahony et $\mathrm{al}^{3}$ suggested that using MDRs is an effective method of reducing length of stay. In a 2011 study, Pacheco et $\mathrm{al}^{4}$ described a statistically significant association of MDRs with reduced length of stay in the intensive care unit. To our knowledge, a study evaluating the effects of daily care coordination on length of hospital stay for patients with chronic obstructive pulmonary disease (COPD) exacerbation in communitybased hospitals has not been published.

The current study included a community hospital that used a daily integrated care conference (ICC) approach. These brief daily ICCs included a participant from each health care provider team involved in a given patient's care and were designed to address any potential barriers in the treatment plan. The goal of these meetings was to organize a systematically coordinated method of communication among health care providers to facilitate a safe and timely discharge. Health care providers included the primary physician group, pharmacists, respiratory therapists, nutritionists, physical and occupational therapists, social workers, and case managers. The ICCs were conducted by a nursing supervisor, typically took place 3 hours after the morning MDRs, and included a representative from each care provider team.

At teaching hospitals, MDRs are often directed by the attending physician with the goal of establishing a decisive diagnosis and treatment plan, whereas ICCs are systematically organized and conducted by the nursing supervisor with the goal of discharge planning in mind. Furthermore, although representatives from several health care provider teams participate in the daily MDRs, their perception and input in the care may be limited. For example, at large teaching institutions where MDRs include at least 10 to 15 people, lack of space at the bedside may limit active participation of each health care provider. In contrast, daily ICCs take place in a conference room where there are no limitations in space. Daily MDRs focus on discovering the correct diagnosis and treatment plan, which may limit important input from care providers who are not physicians. However, daily ICCs take place after MDRs, and other care providers may be more willing to vocalize their input about the patient's care.

The aim of the current study was to determine whether daily ICCs would significantly reduce the length of stay for patients with chronic obstructive pulmonary disease (COPD) exacerbation.

\section{Methods}

Institutional review board approval was obtained from University Hospitals Cleveland Medical Center for a retrospective study of 2 osteopathic community hospitals located in northeast Ohio.

The 2 hospitals were selected based on the presence or absence of ICCs. The hospitals in the study are part of the same hospital system with patient populations of similar socioeconomic status $(\$ 39,000-\$ 43,000 /$ year $)$ and are both located in northeast Ohio. The hospitals' primary care teams are staffed by the same residency training programs and medical students, with some faculty practicing at both locations. Both hospitals have the same ancillary staff available. The number of people without health insurance was less than $10 \%$ at each hospital and within $1 \%$ of each other. The percentage of bachelor-level college education at each township was within $10 \%$ of each other. The study consisted of men and women, aged 40 years and older, who were admitted for no less than 1 day for COPD exacerbations between January 2011 to December 2016 (Figure 1). Race and ethnicity were not tracked in this study. The eligible study population was identified using specific International Classification of Diseases codes within the electronic medical record system: 491.1, 491.2, 491.21, 491.22, 492.21, 492.8, 493.2, 518.81, 770.2, J43.1, J43.2，J43.8，J43.96，J44，J44.1，J44.9，J68.4， J98.2， 


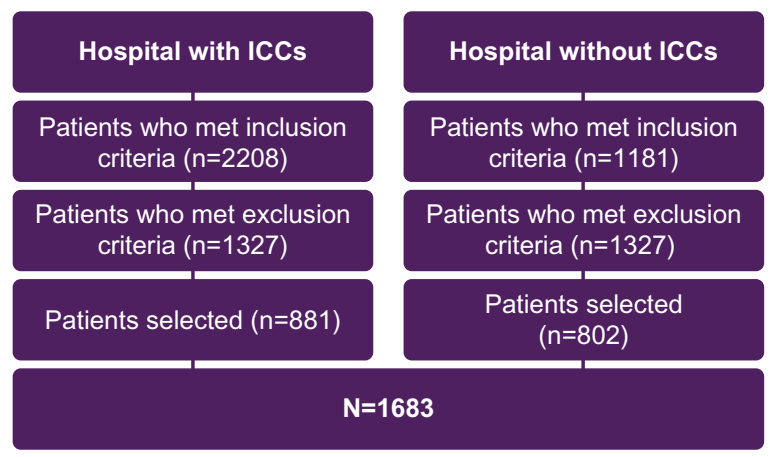

Figure 1.

Flowchart showing patient selection process from 2 community hospitals—one that used integrated care conferences (ICCs) and one that did not use ICCs.

J98.3, P25.0, T79.7, and T81.82. Patients were excluded if they had any of the following known or suspected comorbid conditions: permanent tracheostomy, heart failure with significant left ventricular dysfunction, febrile temperature $\left(>38^{\circ} \mathrm{C}\right)$, pregnancy, lactation, or severe mental illness.

Integrated care conferences were conducted by a nursing supervisor and typically took place 3 hours after morning rounds, with the nursing supervisor verifying that a representative from each health care provider team (ie, primary physician group, pharmacy, respiratory therapy, nutrition, physical and occupational therapy, social work, and case management) was in attendance. The ICC session took place in a quiet conference room with adequate space for all in attendance. The ICCs began with the nursing supervisor presenting the patient individually by his or her name and room number. The participating physician was then asked to provide the working diagnosis, treatments received, disposition, and potential barriers to discharge. Depending on the nature of the barriers to discharge, the nursing supervisor then engaged the appropriate team members in a brief but coordinated discussion to help facilitate a safe and timely discharge.

A 2-tailed $t$ test was used to determine whether there was a statistically significant difference in the average length of stay between the 2 groups. The patients were further age- and gender-matched to aid in controlling for comorbid factors common at different ages and genders. Ages were grouped by decade: 40, 50, 60, 70, 80, and 90 years. In one case, a patient older than 100 years was placed in the 90 -year group. This addition did not alter the statistical significance or trends reported. A 2-tailed $t$ test for analysis of age and sex factors at and between each hospital was conducted. An analysis of variance (ANOVA) was used on the age groups. The Tukey test was performed on the ANOVA of combined age groups as well as at each hospital independently. A $\chi^{2}$ test for trend was used to examine the length of stay between the 2 hospitals. All hospital stays longer than 10 days were put into a separate category (ie, $11+$ ) for the $\chi^{2}$ test because of the randomness of the length of stay after 10 days. All statistics were calculated with $95 \%$ CIs using Prism 7 (GraphPad), and a $P$ value $<.05$ was considered significant.

\section{Results}

There were 3389 eligible patients based on the inclusion criteria (Figure 1). A total of 1706 were removed based on the exclusion criteria, the most common reasons being 0 hospital days and unspecified length of stay (1327 and 379, respectively).

A total of 1683 patients were ultimately selected for data analysis. The group from the hospital with ICCs had 881 patients (372 men and 509 women; Figure 1, 
Table 1.

General Demographics of Patients With COPD at Hospitals With and Without Daily ICCs (N=1683)

\begin{tabular}{|c|c|c|c|c|c|c|c|}
\hline \multirow[b]{2}{*}{ Characteristics } & \multicolumn{3}{|c|}{ Hospital With ICCs, n } & \multicolumn{3}{|c|}{ Hospital Without ICCs, n } & \multirow[b]{2}{*}{ Combined Totals, $n$} \\
\hline & Total & Female & Male & Total & Female & Male & \\
\hline Participants, n & 881 & 509 & 372 & 802 & 488 & 314 & 1683 \\
\hline Mean Age, y & 71.0 & 70.5 & 71.5 & 72.6 & 72.4 & 72.7 & $71.5^{\mathrm{a}}$ \\
\hline 40 Age Group $^{b}$ & 70 & 49 & 21 & 50 & 26 & 24 & 120 \\
\hline 60 Age Group $^{b}$ & 196 & 115 & 81 & 142 & 86 & 56 & 338 \\
\hline 70 Age Group $^{b}$ & 198 & 112 & 86 & 173 & 83 & 90 & 371 \\
\hline 80 Age Group $^{b}$ & 209 & 121 & 88 & 213 & 144 & 69 & 422 \\
\hline
\end{tabular}

a $P=.388$, unpaired 2-tailed $t$ test with $95 \% \mathrm{Cl}$ between hospitals and ages of participants.

b Patients were grouped by decade of life.

Abbreviation: COPD, chronic obstructive pulmonary disease; ICC, integrated care conference.

Table 1). The group from the hospital without ICCs had 802 patients (314 men and 488 women; Figure 1, Table 1).

A 2-tailed $t$ test showed a statistically significant lower length of stay in the hospital with ICCs $(P<.0001)$ (Figure 2A). The mean (SD) length of stay for the hospital with ICCs was 3.37 (2.89) days compared with 5.55 (3.99) days in the hospital without ICCs (Table 2A). When gender matching, the length of stay between the 2 hospitals was also found to be significant $(P<.0001)$ (Table 2A). At the hospital with ICCs, the mean (SD) length of stay was 3.11 (2.34) and 3.56 (3.97) for men and women, respectively. The hospital without ICCs had a mean (SD) length of stay of 5.54 (4.03) and 5.56 (3.23) for men and women, respectively.

A gender comparison of length of stay using a $t$ test was not significant when data for the 2 hospitals were combined ( $P=.08$ ) (Table 2A). However, when comparing gender and length of stay at each hospital independently, the hospital with ICCs did reach statistical significance $(P=.02)$ compared with the hospital without ICCs $(P=.95)$ (Table 2A). However, the statistical significance of the gender comparison at the hospital with ICCs was not found when the genders were age-matched (Table 2B). Using a $t$ test, age comparison between each hospital was also not statistically significant $(P=.39)$.

Age grouping totals were: 120 (40-49 years), 259 (50-59 years), 338 (60-69 years), 371 (70-79 years), 422 (80-89 years), and 173 ( $\geq 90$ years) (Table 1). Separating location and genders found similar trends to the total number of individuals with the highest number of admissions (generally in the 80-89 year group). The only exception was with men in the 70 to 79 year age group making up the largest age subpopulation at the hospital without ICCs. An ANOVA of admission days of all the male patients by age group at the hospital without ICCs was not statistically significant. $(P=.36)$ (Table 3). In contrast, all other ANOVA of admission days at each hospital's totals and genders was found to be statistically significant, ranging from $P=.015$ to $P<.0001$ (Table 3). With the combined data of both hospitals, the length of stay was significantly increased for patients older than 69 years (Figure 2B). This increase was less pronounced at the hospital without ICCs (Figure 2D), particularly 


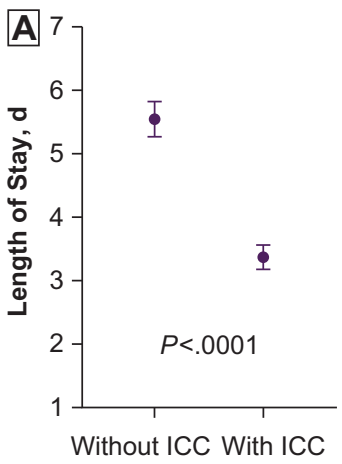

ICC Status

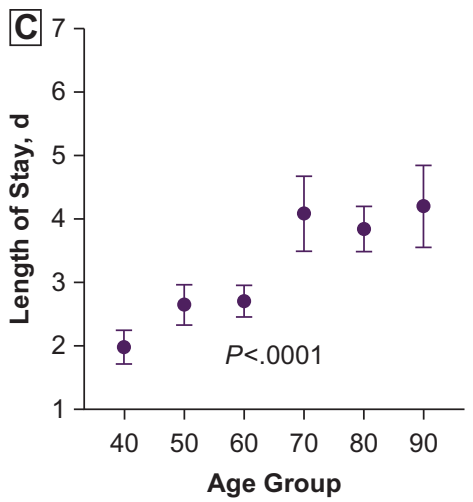

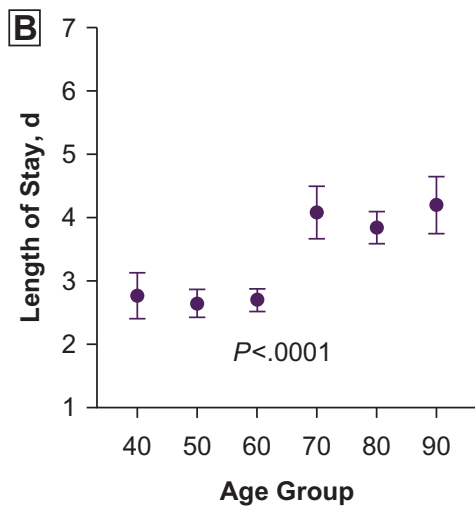

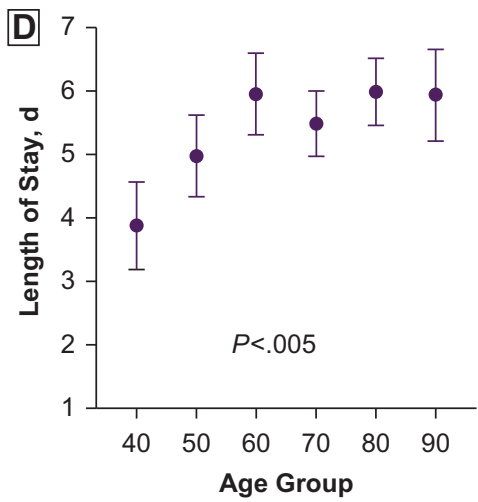

Figure 2.

(A) Mean length of stay between the hospital with integrated care conferences (ICCs) and the hospital without integrated care conferences (unpaired $t$ test; $95 \% \mathrm{Cl})$. (B-D) Analysis of variance $(95 \% \mathrm{Cl})$ of the age groups and the length of stay: (B) combined between the 2 hospitals, (C) the hospital with an ICC, and (D) the hospital without an ICC.

in the cohort of men where there was a noticeable increase in length of stay after the age of 49 years, which contributed to the loss of statistical significance (Table 3). The hospital with ICCs had $61 \%$ to $75 \%$ reduction in length of stay for the ages 40 to 69 years. This trend continued for the patients aged 70 years or older, but the percent difference was not as pronounced and ranged from $29 \%$ to $44 \%$.

Table 2A.

Gender- and Age-Matched Unpaired $t$ Test and Average Length of Stay at Hospitals With and Without Daily Integrated Care Conferences $(\mathrm{N}=1683)^{\mathrm{a}}$

\begin{tabular}{lcccr} 
Gender & Hospital & P Value & \multicolumn{2}{c}{$\begin{array}{c}\text { Mean (SD) of Length of Stay } \\
\text { (Gender) }\end{array}$} \\
\hline Both vs Both & Both & $<.0001$ & $3.37(2.89)^{\mathrm{b}}$ & $5.55(3.99)^{\mathrm{c}}$ \\
\hline Female vs Male & Both & .078 & $4.54(3.74)(\mathrm{F})$ & $4.22(3.44)(\mathrm{M})$ \\
\hline Female vs Male & With ICCs & .021 & $3.56(3.23)(\mathrm{F})$ & $3.11(2.34)(\mathrm{M})$ \\
\hline Female vs Male & Without ICCs & .95 & $5.55(4.03)(\mathrm{F})$ & $5.54(3.97)(\mathrm{M})$ \\
\hline
\end{tabular}




\section{Table 2B.}

\begin{tabular}{|c|c|c|}
\hline \multirow{2}{*}{$\begin{array}{l}\text { Patient } \\
\text { Female vs } \\
\text { Male }^{\text {d }}\end{array}$} & \multicolumn{2}{|c|}{$P$ Value } \\
\hline & $\begin{array}{l}\text { Hospital With } \\
\text { ICCs }\end{array}$ & $\begin{array}{l}\text { Hospital Without } \\
\text { ICCs }\end{array}$ \\
\hline 40 & .12 & .064 \\
\hline 50 & .41 & .70 \\
\hline 60 & .050 & .32 \\
\hline 70 & .13 & .42 \\
\hline 80 & .27 & .91 \\
\hline 90 & .25 & .59 \\
\hline
\end{tabular}

a All $t$ tests are unpaired, 2-tailed, and all statistical calculations are done with $95 \% \mathrm{Cl}$

b Patients at hospitals with integrated care conferences (ICCs).

Patients at hospitals without ICCs.

d Age group indicates the general decade of life.

A $\chi^{2}$ test for trend of all the length of stays at each hospital yielded $P<.0001\left(\chi^{2}=201.5\right)$ (Table 3). The hospital with ICCs had a significantly larger number of discharges prior to the fourth day of the hospital stay compared with the hospital without ICCs (Figure 3A). This was true for the age groups of 40 to 69 years and 70 to 99 years and older as well (Table 3, Figure 3B and 3C). At hospital stays of 5 days and longer, the hospital without ICCs had a higher number of patients still admitted regardless of the age group. After day 5, of the 2 hospitals combined, the percentage of patients from the hospital with ICCs that made up the total patients still admitted ranged from about $13 \%$ to $30 \%$, with $25 \%$ being the average. However, the hospital with ICCs had the longest hospital stay of the 2 hospitals (39 days vs 31 days).

\section{Discussion}

Chronic obstructive pulmonary disease is a manageable condition that most commonly presents with symptoms of cough, shortness of breath, and sputum production. Smoking is the most common risk factor
Table 3.

ANOVA and $\chi^{2}$ for Trend Data Among Patients With COPD at Hospitals With and Without Daily ICCs $(\mathrm{N}=1683)^{\mathrm{a}}$

\begin{tabular}{|c|c|}
\hline Hospital (Gender) & P Value \\
\hline With ICCs (female) & $<.0001$ \\
\hline With ICCs (male) & $<.0001$ \\
\hline Without ICCs (female) & .015 \\
\hline Without ICCs (male) & .36 \\
\hline \multicolumn{2}{|c|}{ ANOVA Length of Stay of Age Groups } \\
\hline \multicolumn{2}{|l|}{ Hospital } \\
\hline Both $^{b}$ & $<.0001$ \\
\hline With ICCs ${ }^{b}$ & $<.0001$ \\
\hline Without ICCs ${ }^{\mathrm{C}}$ & .005 \\
\hline \multicolumn{2}{|c|}{$x^{2}$ for Trend: Length of Stay Hospital Comparison ${ }^{d}$} \\
\hline \multicolumn{2}{|l|}{ Length of Stay } \\
\hline All ages & $<.0001$ \\
\hline Age $40-69$ y & $<.0001$ \\
\hline Age $70-99+y$ & $<.0001$ \\
\hline
\end{tabular}

a All statistical calculations were done with $95 \%$ Cls.

b Tukey test the statistically significant difference was between age groups $40-60$ and $70-90$ years.

c Tukey test the statistically significant difference was between age group of 40 years and the age groups of 50 to 90 years.

d Both genders are included and degrees of freedom for entries are all ages, 201.5; ages 40-69 years, 128.2; and ages 70-99+ years, 81.22 .

Abbreviations: ANOVA, analysis of variance; ICCs, integrated care conferences.

for COPD; however, environmental exposures and genetic and developmental abnormalities also contribute to its pathogenesis. Chronic obstructive pulmonary disease is also the third leading cause of death in the United States, and it is associated with economic costs of approximately $\$ 50$ billion annually. ${ }^{7}$ Hospital care and emergency department visits related to COPD exacerbation contribute to $70 \%$ of COPD-related health expenses. ${ }^{7,8}$ Methods are needed to help decrease the costs attributed to the management of COPD exacerbation provided the standard of care is not compromised. 

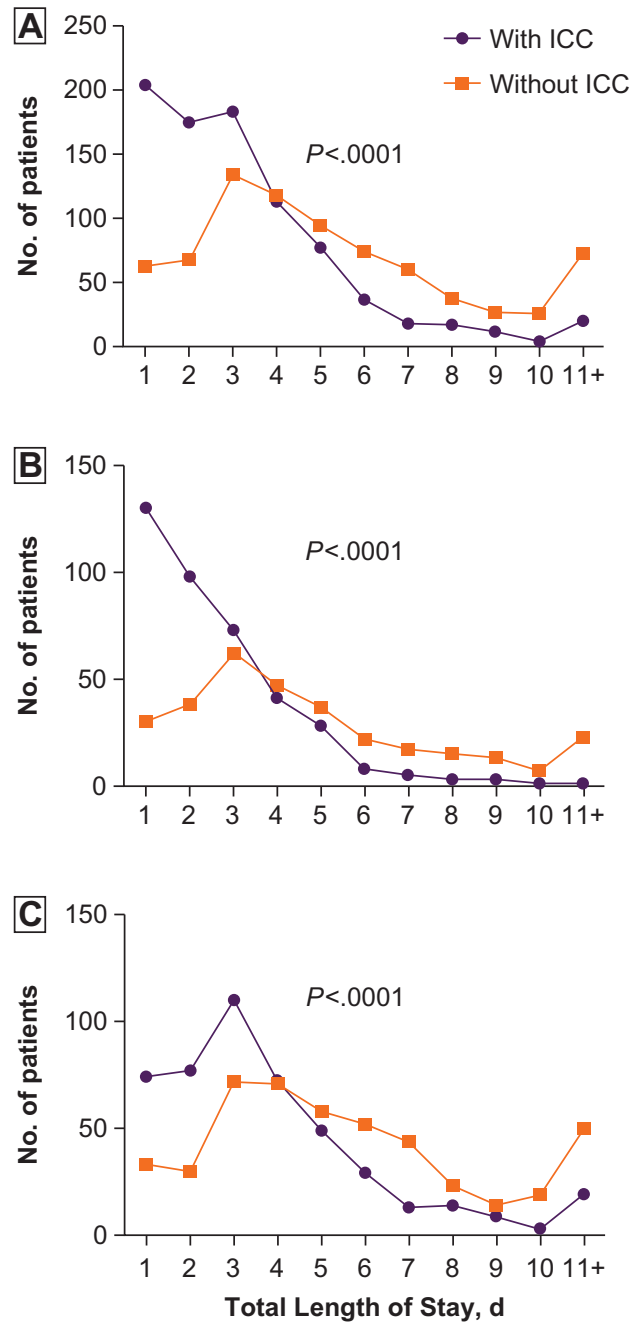

Figure 3.

$\chi^{2}$ for trend with $95 \% \mathrm{Cl}$ and the number of patients and the respective length of stay in the hospital. (A) All age groups; (B) ages 40-60 years; (C) ages 70-90 years.

Abbreviation: ICC, integrated care conference.

Additional cost to the health care system related to COPD exacerbation in recent years is felt, in part, to be from an unnecessary increase in length of hospital stay, which may be caused by a health care system that is increasingly complex across all disciplines and staffed in an environment riddled with burnout. ${ }^{9}$ Creative and integrated approaches to care coordination must replace the traditional models in order to counteract this mounting complexity and exhaustion. ${ }^{10}$
It is worth noting that this project was initiated by residents' observation of the efficiency of the ICCs with respect to workload and ease of communication. Length of stay was chosen for investigation because if the sentiments noted by the doctors-in-training were true, the increase in efficient communication and work throughput might be reflected in an average decrease in length of stay between a hospital that has daily ICCs and a hospital that does not.

The average length of stay was much lower at the hospital with ICCs. This was true overall as well as with respect to each age group as supported by the ANOVA results. A Tukey test showed the statistical significance was between the age groups of 40 to 60 and 70 to 90 years. There was no statistical significance between the age groups of 40 to 60 years and the age groups of 70 to 90 years at the hospital with ICCs. However, the Tukey test showed that there was statistical significance between the age group of 40 years and the age groups of 50 to 90 years at the hospital without ICCs. There was no statistical significance at the hospital without ICCs between age groups of 50 to 90 years. However, there was a considerable drop in the percent difference in earlier discharges between the 2 hospitals when analyzing the patients aged 40 to 69 years. These findings are consistent with the literature in that recovery times are longer for patients as they get older and acquire more comorbid health conditions. $^{11}$

As the length of stay increased because of the need for inpatient care, the usefulness of the ICC approach became less pronounced. This could be attributed to time not being the limiting factor in the facilitation of communication and coordinating care for discharge as the patients recovered. This trend suggests that the needs of patient care are governing the length of stay for patients who are more frail rather than a discharge agenda. There does still seem to be some benefit, albeit attenuated, favoring ICCs in reducing length of stay even in older patients. Although the average length of stay was lower at the hospital with ICCs, the longest stay noted in this study was at the hospital 
with ICCs (woman, aged 71 years; length of stay, 39 days). This finding is evidence that care needs were not being compromised.

Daily ICCs provide for a holistic, whole-person approach to treating patients with COPD exacerbation who are in the hospital and reflect the tenets of osteopathic medicine. ${ }^{11}$ The ICC approach also addresses the collaborative and comprehensive communication among multiple health care providers.

There are often unique comorbid illnesses between the genders, and gender matching helps to address this potential confounder. ${ }^{10}$ With gender matching, the length of stay was shorter at the hospital with ICCs between the 2 locations. Gender comparison at the hospital with ICCs showed a statistical significance. The statistical significance of the gender comparison at the hospital with ICCs overall did not hold up to the agematching analysis. Additionally, the longest hospital stay for men at the hospital with ICCs was 17 days; there were 3 women with hospital stays of 21, 32, and 39 days. The elimination of the latter 2 as outliers by the rationale of the Grubbs test resulted in a loss of statistical significance $(P=.05)$.

The hospital with ICCs discharged more patients aged 40 to 69 years within the first 3 days of hospital stay than the hospital without ICCs. Although the hospital with ICCs discharged more patients aged 70 years and older, the pattern of discharges peaked at day 3 for both hospitals for this patient cohort. There was also a noticeable decrease in the difference between days 1 and 2 compared with other age cohorts. This finding shows that with increased frailty, the length of stay was more likely to be longer regardless of implementing ICCs or not. It is likely that as the length of stay increases, the advantage of ICCs decreases because there is more time to address coordinating care.

A limitation of this study was that its retrospective nature made controlling for the exact disease severity, comorbid conditions, and treatment plans problematic and more appropriate for a prospective study. Some of the strengths of this study were its sample size at each location, similarly paired osteopathic community-based hospitals within the same hospital system and electronic medical record, as well as the partially shared staffing by way of the doctors-in-training. Each aspect helped mitigate the confounding errors that were hard to control for in a retrospective study.

The inference that decreased communication results in increased length of stay and vice versa for a patient is due to the time it takes for the primary team to receive help from consultations and ancillary staff. The hospital primary team often relies on this input and assistance to treat and discharge a patient with COPD in a safe and timely manner. For example, it is not uncommon for a patient with COPD to be cared for by specialty consultants, pharmacists, respiratory therapists, dieticians, physical and occupational therapists, social workers, and case managers. The notion that lack of a systematically coordinated method of communication among care providers can lead to delayed care or execution of an appropriate care plan resulting in an increased length of stay is supported by the data found in this study.

\section{Conclusion}

This study found a statistically significant reduction in length of hospital stay for patients with COPD exacerbations by means of an ICC model. The dichotomies were most pronounced within the first 3 days of hospital stay and in patients aged 40 to 69 years. The reduced length of stay was true for all ages and to a certain extent for the number of patients hospitalized for longer than 4 days. The implementation of ICCs has the potential to make health care services and coordinated care more efficient and result in decreased cost related to the length of stay. Additional studies of patients with other disease processes are needed to further evaluate the effectiveness of ICCs in reducing length of hospital stay.

\section{Acknowledgments}

We acknowledge Pauline Yurchenko, MEd, for her encouragement and administrative support during the progression of this manuscript. We also thank Janet Peachey, MPH, for her help with preparation of this manuscript for publication and Nikki Niemeyer, MA, for her assistance with data analysis. 


\section{Author Contributions}

All authors provided substantial contributions to conception and design, acquisition of data, or analysis and interpretation of data; all authors drafted the article or revised it critically for important intellectual content; all authors gave final approval of the version of the article to be published; and all authors agree to be accountable for all aspects of the work in ensuring that questions related to the accuracy or integrity of any part of the work are appropriately investigated and resolved.

\section{References}

1. Plsek PE, Greenhalgh T. Complexity science: the challenge of complexity in health care. BMJ. 2001;323(7313):625-628. doi:10.1136/ bmj.323.7313.625

2. Gruenberg DA, Shelton W, Rose SL, Rutter AE, Socaris S, McGee G. Factors influencing length of stay in the intensive care unit. Am J Crit Care. 2006;15(5):502-509.

3. O'Mahony S, Mazur E, Charney P, Wang Y, Fine J. Use of multidisciplinary rounds to simultaneously improve quality outcomes, enhance resident education, and shorten length of stay. J Gen Intern Med. 2007;22(8):1073-1079. doi:10.1007/s11606-007-0225-1

4. Pacheco ES, Campos IP, Seixas JF, et al. Daily multidisciplinary rounds reduce ICU length of stay. Crit Care. 2011;15(suppl 2):P53 doi:10.1186/cc10201

5. Peppers BP, Varma P, Kim YM, Hostoffer JR, Rowane MP. Scholar 7: The development of regional community hospitals' scholastic environment. J Am Osteopath Assoc. 2017;117(10):643-650. doi:10.7556/jaoa.2017.121

6. Guarascio AJ, Ray SM, Finch CK, Self TH. The clinical and economic burden of chronic obstructive pulmonary disease in the USA. Clinicoecon Outcomes Res. 2013;5:235-245. doi:10.2147/CEOR.S34321

7. Vogelmeier C, Criner GJ, Martinez FJ, et al. Global initiative for chronic obstructive lung disease (GOLD) revisions 2001-2017: historical and critical perspective. Am J Respir Crit Care Med. 2017;195(5):557-582. doi:10.1164/rccm.201701-0218PP.

8. Dewa CS, Loong D, Bonato S, Trojanowski L. The relationship between physician burnout and quality of healthcare in terms of safety and acceptability: a systematic review. BMJ Open. 2017;7(6):e015141. doi:10.1136/bmjopen-2016-015141

9. Anderson LA, Goodman RA, Holtzman D, Posner SF, Northridge ME. Aging in the United States: opportunities and challenges for public health. Am J Public Health. 2012;102(3):393-395. doi:10.2105/ AJPH.2011.300617

10. DiGiacomo M, Green A, Rodrigues E, Mulligan K, Davidson PM. Developing a gender-based approach to chronic conditions and women's health: a qualitative investigation of community-dwelling women and service provider perspectives. BMC Womens Health 2015;15(1):105. doi:10.1186/s12905-015-0264-4

11. Tenets of osteopathic medicine. American Osteopathic website. https://osteopathic.org/about/leadership/aoa-governance-documents/ tenets-of-osteopathic-medicine. Accessed February 13, 2020.

(c) 2020 American Osteopathic Association 